\title{
Hirudotherapy - a rare cause of pseudolymphoma
}

\author{
Małgorzata Tupikowska ${ }^{1}$, Zdzisław Woźniak², Marta Wojciechowska-Zdrojowy' ${ }^{1}$, Joanna Maj ${ }^{1}$, \\ Alina Jankowska-Konsur ${ }^{1}$
}

${ }^{1}$ Department of Dermatology, Venereology and Allergology, Wroclaw Medical University, Wroclaw, Poland ${ }^{2}$ Department of Pathomorphology, Wroclaw Medical University, Wroclaw, Poland

Adv Dermatol Allergol 2018; XXXV (2): 225-226

DOI: https://doi.org/10.5114/ada.2018.75250

Cutaneous pseudolymphoma (C-PSL) represents a heterogeneous group of cutaneous reactions characterized by polyclonal T and/or B cell proliferation [1, 2]. The disease can be either idiopathic or triggered by various stimuli including infections, primarily Borrelia burgdorferi and drugs $[3,4]$. Several lines of evidence indicate minor traumas such as tattoos, acupuncture, insect bites and vaccination as C-PSL inducers [3, 4]. Herein, we present a rare case of a C-PSL provoked by hirudotherapy.

A 38-year-old woman was admitted to the Department of Dermatology, Venereology and Allergology, Wroclaw Medical University, for diagnosis and treatment of skin lesions that appeared 7 months before hospitalization. On admission, physical examination revealed 10 well-defined, firm and pruritic nodules, red in color, ranging from 6 to $10 \mathrm{~mm}$ in diameter in her pubic area (Figures $1 \mathrm{~A}, \mathrm{~B}$ ). The skin changes appeared soon after the patient underwent hirudotherapy that had been recommended by her friend as a successful treatment for uterine myoma. The pruritic cutaneous lesions appeared after the first procedure, however the patient decided to apply the second therapy after 3 weeks. Each time 5 leeches were used. For the apparent skin lesions the patient was given oral antihistamines and moderate potent topical glucocorticosteroids with no improvement. The patient did not take any other medicines, except for ulipristal acetate $5 \mathrm{mg}$ /day, orally, administered by a gynecologist for the uterine myoma.

Laboratory tests (morphology, urinalysis, lipids, liver function, renal function and inflammation markers) were within normal ranges. Histological examination of the skin biopsy revealed irregular acanthosis and mixedcell infiltration, composed of lymphocytes, histiocytes, plasma cells and eosinophils in the dermis. An evident exocytosis with focal spongiosis and intraepidermal inflammatory infiltration were observed. In the immunohistochemical study, the lymphocyte population consisted of a mixed population of $\mathrm{T}$ and $\mathrm{B}$ lymphocytes with
T cell predomination. The T lymphoid cells were positive for CD3, CD43, CD45RO, CD4, CD8, CD30, CD20, CD79a, CD138. The numerous epithelioid histiocytes were CD68 positive. The proliferative rate measured by Ki-67 expression was low (labeling index $=7 \%$ ). Based on the histological, immunohistochemical results and clinical observations the diagnosis of C-PSL was made.

Initially, the skin changes were treated with $100 \mathrm{mg}$ hydrocortisone intralesionally and topical glucocorticosteroid ointment (clobetasol propionate) applied twice a day. Cryotherapy was used in the case of one nodule, however the results were unsatisfactory as the nodule became discolored. After 4 weeks of topical treatment, nodules became smaller and less red but remained firm. We modified the previous therapy by adding methylprednisolone acetate intramuscularly $40 \mathrm{mg}$ every 4 weeks. We chose intramuscular instead of intralesional drug administration because the patient had a bad experience with previous mesotherapies and preferred systemic treatment (one injection) instead of a series of injections. The patient continued treatment for 20 weeks with a slow regression of skin lesions.

Cutaneous pseudolymphomas are not a rare dermatological problem, however, due to the overlapping histopathological and clinical features they may create a diagnostic and therapeutic challenge even for experienced dermatologists $[5,6]$. To diagnose C-PSL, histological and clinical criteria must be fulfilled [5, 6]. Clinical criteria include typical location and morphology of the skin changes as well as characteristic clinical course of the disease. Typically, skin changes appear on the face, which is the most common localization of the lesions, on the chest and upper extremities. They are usually single and localized nodules or plaques, red to purple in color and tend to be self-regressing or disappear when the causative factor is removed [3]. Histological features of C-PSL include polyclonal lymphocyte infiltration in the upper layers of the skin. According to the predominant

Address for correspondence: Alina Jankowska-Konsur MD, PhD, Department and Clinic of Dermatology, Venereology and Allergology, Wroclaw Medical University, 1 Chałubińskiego St, 50-368 Wroclaw, Poland, phone: +48 7178422 86, +48 691 033632 , fax: +48 7132709 42, e-mail: alina.jankowska-konsur@umed.wroc.pl Received: 29.03.2017, accepted: 21.04.2017. 

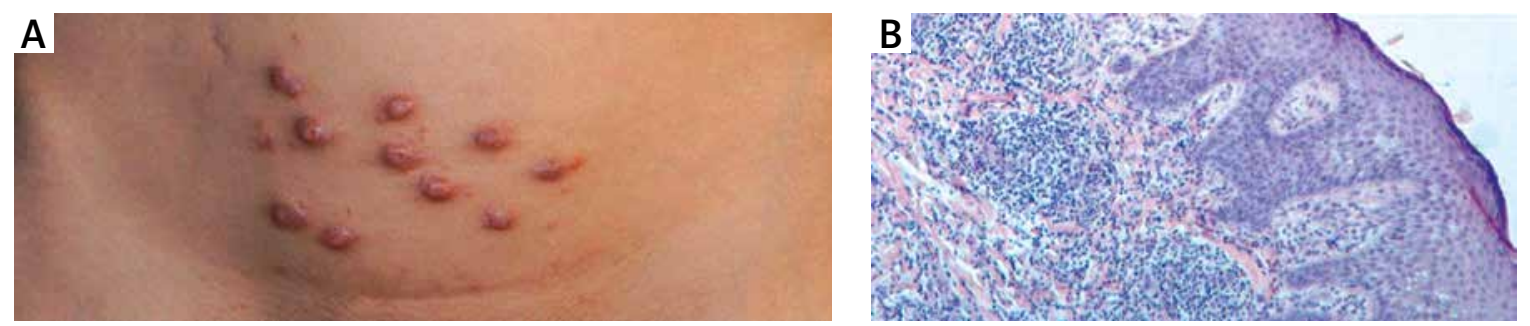

Figure 1. A - A 38-year-old female with skin changes in the pubic area after hirudotherapy. B - A significant inflammatory infiltration in the dermis composed mostly of lymphocytes and eosinophils. An evident epidermotropism with spongiosis was observed in some parts of the infiltration (hematoxylin and eosin, magnification 100x)

lymphocyte type in the infiltrate, several types of cutaneous pseudolymphomas have been distinguished: C-PSL with predominant T-cell (T-PSL), B-cell (B-PSL) and mixed cell infiltrations. C-PSLS with mixed cell infiltration, as in our case, usually occur after acupuncture, vaccination, tattoos and in Borrelia burgdorferi infections. T-PSL infiltration was reported as the effect of persistent scabies, chronic contact dermatitis, arthropod bites, drug reaction and in idiopathic cases [7]. B-PSLs are often related to drug-induced reactions [8].

Hirudotherapy has been used in the traditional medicine for bloodletting for medical conditions such as chronic venous insufficiency and muscular pain as well as cosmetic reasons. The complications of the procedure include infections, local irritation, scarring, bleeding and anemia. To the best of our knowledge, this is the first case of pseudolymphoma after hirudotherapy reported in Poland, and there have been only three such cases described in the world literature [9-11]. In the published reports medicinal leeches were applied for fibromyalgia, chronic venous insufficiency with varicosis, and, in 1 case, for cosmetic reasons (black circles in the infraorbital regions). In contrast to our case, where we found mixed $B$ and T-cell infiltrate in the skin biopsy, the biopsies taken from the skin lesions in the reported cases revealed predominant $B$-cell infiltrates in two cases and T-cell infiltrate in 1 case. Topical and intralesional glucocorticosteroids, antibiotics, ultraviolet B phototherapy, cyclosporine $A$, interferon $\alpha 2 \beta$, laser therapy and surgical excision have been reported as a successful therapy of the pseudolymphomas [12]. The reported cases of pseudolymphomas after medical leeches were treated successfully with either topical mometasone furoate or intralesional triamcinolone acetonide injections with complete clearing of the lesions [9-11].

In recent years there has been increased interest in alternative methods of treatment, including hirudotherapy. However, we should always keep in mind undesirable side effects of these procedures such as development of pseudolymphoma.

\section{Conflict of interest}

The authors declare no conflict of interest.

\section{References}

1. Bergman R. Pseudolymphoma and cutaneous lymphoma: facts and controversies. Clin Dermatol 2010; 28: 568-74.

2. Sander CA. Pseudolymphoma. In: Braun Falco Dermatologia. Burgdorf WHC, Plewig G, Wolff HH, Landthaler M (eds.). Czelej, Lublin 2011; 1502-6.

3. Moniuszko A, Czupryna P, Pancewicz S, et al. Borrelial lymphocytoma: a case report of a pregnant woman. Ticks Tick Borne Dis 2012; 3: 257-8.

4. Koh WL, Tay YK, Koh M, et al. Cutaneous pseudolymphoma occurring after traumatic implantation of a foreign red pigment. Singapore Med J 2013; 54: e100-1.

5. Terada T. Cutaneous pseudolymphoma: a case report with an immunohistochemical study. Int J Clin Exp Pathol 2013; 6: 966-72.

6. Kerl H, Fink-Puches R, Cerroni L. Diagnostic criteria of primary cutaneous B-cell lymphomas and pseudolymphomas. Keio J Med 2001; 50: 269-73.

7. Marchesi A, Parodi PC, Brioschi M, et al. Tattoo ink-related cutaneous pseudolymphoma: a rare but significant complication. Case report and review of the literature. Aesthetic Plast Surg 2014; 38: 471-8.

8. Kitagawa KH, Grassi M. Zoledronic acid-induced cutaneous B-cell pseudolymphoma. J Am Acad Dermatol 2011; 65: 1238-40.

9. Altamura D, Calonje E, Liau J, et al. Diffuse cutaneous pseudolymphoma due to therapy with medicinal leeches. JAMA Dermatol 2014; 150: 783-4.

10. Choi Y, Kim SC. Cutaneous pseudolymphoma induced by Hirudo medicinalis therapy. J Dermatol 2012; 39: 195-7.

11. Smolle J, Cerroni L, Kerl H. Multiple pseudolymphomas caused by Hirudo medicinalis therapy. J Am Acad Dermatol 2000; 43: 867-9.

12. Tomar S, Stoll H, Grassi M, et al. Treatment of cutaneous pseudolymphoma with interferon alfa-2b. J Am Acad Dermatol 2009; 60: 172-4. 